\title{
Bei Sportlern ist die Ablation Therapie der Wahl
}

\author{
Sport und Vorhofflimmern. Sicherlich ein brisantes Thema mit vielen \\ Fragen! Haben Leistungssportler ein erhöhtes Risiko und wie sollte \\ man sie behandeln? Und ist Sport für den Patienten mit paroxysmalem \\ oder permanentem Vorhofflimmern schädlich oder günstig?
}

- Das Auftreten von Vorhofflimmern bei Sportlern bedeutet für die Betroffenen meist eine deutliche Abnahme ihrer Lebensqualität, zumal durch die Rhythmusstörung auch ihre sportliche Leistungsfähigkeit beeinträchtigt wird. „Dazu kommt, dass die antiarrhythmische Therapie mit einer Reihe von Problemen bzw. Limitationen assoziiert ist", erläuterte Prof. Wilhelm Haverkamp von der kardiologischen Universitätsklinik der Charité in Berlin. Wegen der verstärkten Bradykardie-Neigung werden Betablocker häufig schlecht vertragen und sie vermindern auch die körperliche Leistungsfähigkeit. Außerdem stehen diese Substanzen zumindest teilweise auf der Dopingliste. Und beim Einsatz von Klasse-I-Antiarrhythmika besteht aufgrund der frequenzabhängigen $\mathrm{Na}$ Blockade ein erhöhtes Proarrhythmierisiko. „Angesichts dieser vielfältigen Probleme ist bei Sportlern heute die Katheterablation die Therapie der ersten Wahl“, so Haverkamp.

\section{Erhöht Sport das Risiko?}

In diesem Zusammenhang wird auch immer wieder darüber diskutiert, ob eine intensive sportliche Betätigung das Risiko für das Auftreten von Vorhofflimmern erhöht. „Es gibt in der Tat Studien, die zeigen, dass Vorhofflimmern gehäuft bei Sportlern, vor allem bei älteren Sportlern auftritt“, so Haverkamp. Betroffen seien vorwiegend Ausdauer sportler wie Marathonläufer. Auch habe sich eine positive Korrelation zwischen der Vorhofflimmern-Inzidenz und der sportlichen Gesamtleistung gezeigt. So war das Risiko erst dann - und zwar um das fast Dreifache - erhöht, wenn mehr als 1.500 Stunden Sport im Leben geleistet wurden. Darunter war die Vorhofflimmern-Inzidenz allerdings nicht erhöht. „Vieles spricht dafür, dass es Personen trifft, die auch ohne Sport Vorhofflimmern bekommen hätten, aber der Sport führt dazu, dass Vorhofflimmern früher auftritt", so Haverkamp.

\section{Sport verbessert die körperliche Leistungsfähigkeit}

Doch sollten deshalb Patienten mit Vorhofflimmern gar keinen Sport treiben? „Keinesfalls, denn bei Patienten mit paroxysmalem Vorhofflimmern kann durch ein regelmäßiges moderates Ausdauertraining das Rezidivrisiko sogar gesenkt werden, d. h. die Intervalle zwischen den Vorhofflimmern-Episoden werden länger und die Episoden kürzer“, so Haverkamp. Empfehlenswert sei ein Intervalltraining 3- bis 5-mal wöchentlich mit einer Gesamtdauer von 2 bis 3 Stunden, wobei 95\% der maximalen Herzfrequenz erreicht werden sollte.

Ein solches Training reduziert nicht nur die Vorhofflimmern-Last, sondern verbessert auch die Symptomatik. Zu- sätzlich werden Blutdruck, Blutzucker und Gewicht günstig beeinflusst. Und Patienten, die eine anhaltende Gewichtreduktion erreichen, haben seltener Vorhofflimmern-Rezidive.

Als mögliche Mechanismen für den günstigen Effekt auf die Rhythmusstörung werden eine Rückbildung des atrialen Remodelling, eine Stabilisierung des vegetativen Nervensystems und antientzündliche Effekte diskutiert.

Auch bei einem permanenten Vorhofflimmern ist sportliche Betätigung vorteilhaft; denn durch ein Belastungstraining werden auch bei diesen Patienten die Häufigkeit und die Schwere der Beschwerden reduziert.

Problematisch kann bei solchen Patienten allerdings der sehr rasche Pulsanstieg unter Belastung sein. Deshalb ist eine optimale Frequenzkontrolle mit Betablockern, evtl. in Kombination mit Digitalis, sehr wichtig. In einer Studie konnte mit einem regelmäßigen Training die Wegstecke beim Walking von 1,8 auf 4,0 km und die Dauer der körperlichen Belastung von 24 auf 48 Minuten gesteigert werden.

Dr. Peter Stiefelhagen

- Quelle: 5. Hannover Herz Lungen Messe, 10.3.2017 in Hannover 